\title{
Medication Adherence of Patients with Pemphigus and Bullous Pemphigoid Disorders in a Tertiary Care Teaching Hospital - A Prospective-Observational Study
}

\author{
Jerin James ${ }^{1, *}$, Jayakrishnan Sadasivan', Anuja Elizabeth George ${ }^{2}$ \\ ${ }^{1}$ Department of Hospital and Clinical Pharmacy, College of Pharmaceutical Sciences, Government Medical College, \\ Thiruvananthapuram, Kerala, INDIA. \\ ${ }^{2}$ Department of Dermatology and Venereology, Government Medical College, Thiruvananthapuram, Kerala, INDIA.
}

\begin{abstract}
Background: Pemphigus and Bullous Pemphigoid are auto-immune blistering diseases. Management of these chronic and severe conditions requires various modes of prolonged therapy. Therefore, medication adherence has a very important and essential role in treating these disorders. Objectives: The goal of the research was to assess the medication adherence of patients with Pemphigus and Bullous Pemphigoid and to correlate it with the quality of life of these patients. Methods: A 'prospective, observational' study was conducted in the 'Department of Dermatology and Venereology', in a tertiary care centre from December 2019 to June 2020. A sample of 28 patients was enrolled in the study. Medication adherence was calculated by using the 'Morisky Green Levine medication adherence scale'. Quality of life was evaluated by using 'Dermatology Life Quality Index'. Results: The 28 patients were aged between 24 and 82 years with a mean of $52.61 \pm 15.247$ years. Majority of patients $(82.1 \%)$ belonged to the High Adherence group, while $14.3 \%$ of the patients belonged to the Moderate adherence group and only $3.6 \%$ of patients belonged to the Low medication adherence group. The initial quality of life (DLQI 1) of patients had a mean score of $14.11 \pm 4.508$ and the quality of life of the same patients after 3 months (DLQI 2) had a mean score of $4.86 \pm 4.897$. A statistically significant relationship between the quality of life and adherence to medication was observed in this study. Conclusion: High adherence to medication was seen in most patients.
\end{abstract}

Key words: Pemphigus, Corticosteroid therapy, Quality of life, Medication Adherence, Dermatology Life Quality Index.

\section{INTRODUCTION}

According to WHO, "medication adherence" may be defined as "the degree to which the person's behaviour corresponds with the agreed recommendations from a health care provider". "Adherence signifies that the patient and physician collaborate to improve the patient's health by integrating the physician's medical opinion and the patient's lifestyle, values and preferences for care". ${ }^{2-4}$ Success of any treatment depends on adherence to treatment which in turn affects the health care system. Medication adherence is an essential component for managing chronic diseases and non-adherence leads to worsening of disease or death and thereby, increases the cost of health care. There are a number of published works available about medication adherence in the fields of haemodialysis, oncology, etc, but there is only very limited literature available regarding skin diseases. Therefore, we evaluated the medication adherence
DOI: 10.5530/ijopp.14.4.54

Address for correspondence: Jerin James, M.Pharm, Department of Hospital and Clinical Pharmacy, College of Pharmaceutical Sciences, Government Medical College, Thiruvananthapuram-695011, Kerala, INDIA.

Phone no: +919539059096 Email id: jerin.james@learner. manipal.edu

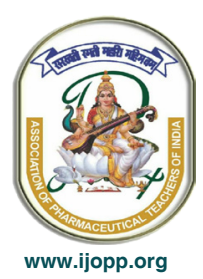


of patients with pemphigus and bullous pemphigoid disorders in the Department of Dermatology and Venereology, in a tertiary care teaching hospital, from December 2019 to June 2020.

The word Pemphigus is derived from the Greek word "Pemphix". Pemphigus and Bullous Pemphigoid are auto-immune life-threatening diseases characterized by vesicles, bullae and erosions involving skin, scalp and mucosae. Blistering may be associated with severe pain. Pemphigus and Bullous Pemphigoid are chronic disorders which do not get cured without treatment. The severity of these lesions can significantly reduce the quality of life.

Medication Adherence is usually assessed using selfreport questionnaires in both developing countries and industrialized, because it is uncomplicated and economical. . "Morisky Green Levine Medication Adherence Scale" (MGLS) is the one of the commonly used self-report questionnaire, a freely available questionnaire with 4 items. ${ }^{6}$ The main concept of the scale is that, Non-adherence of the medication is attributed to negligence, forgetting, discontinuing the medication when feeling improved or quit the treatment when feeling worse. ${ }^{7}$ The MGLS assess both unintended non-adherence (carelessness, forgetting) and intentional non-adherence (stopping the drug when feeling improved or worse). The response is recorded in the style of "dichotomous responses" (yes and no). ${ }^{8}$ The MGLS has been practice in various nations and its validity and reliability is observed to be good enough to assess the adherence in hypertension, ${ }^{9}$ diabetes $^{8}$ and atherosclerosis. ${ }^{6}$

The score is calculated based on the number of 'yes' items. The range of score is $0-4$, " 0 " means high adherence, 1-2 means medium level of adherence and 3-4 means low level adherence (Table 1).

DLQI (Dermatology Life Quality Index) is a questionnaire-based evaluation tool devised by Finlay

Table 1: Morisky Green Levine questionnaire.

\begin{tabular}{cc} 
SI.No & Yes \\
\hline 1 & Do you ever forget your medicine? \\
2 & $\begin{array}{c}\text { Are you careless at times about } \\
\text { taking your medicine? }\end{array}$ \\
3 & $\begin{array}{c}\text { When you feel better, do you } \\
\text { sometimes stop taking your } \\
\text { medicines? }\end{array}$ \\
& When you take medicine, do you \\
& stop taking it? \\
\hline
\end{tabular}

and $\mathrm{Khan}^{10}$ and was suggested as an appropriate tool for evaluating the quality of life of patients with Pemphigus and Bullous Pemphigoid. ${ }^{11}$ The socio-economic status of the patients were evaluated based on the Modified Kuppuswamy's socio-economic scale. ${ }^{12}$ In India, studies regarding the evaluation of medication adherence in autoimmune bullous disorders are very limited, so the current study aims to evaluate the medication adherence of patients with Pemphigus and Bullous Pemphigoid disorder and correlate it with the quality of life of the patients.

\section{MATERIALS AND METHODS}

It was a "Prospective-observational study", Conducted in the Department of "Dermatology and Venereology", in a tertiary care center from December 2019 to June 2020. Informed consent was obtained from Patients with Pemphigus and Bullous Pemphigoid disorder. This is the first study of evaluation of medication adherence in this disorder using the above tool, to the best of our knowledge. Since no reports on medication adherence in case of the autoimmune blistering diseases pemphigus and bullous pemphigoid could be accessed on literature search to base this study to calculate the sample size, continuous sampling was implemented and all cases of pemphigus and bullous pemphigoid satisfying the inclusion criteria, attending the department of Dermatology and Venereology during the study period were included in the study. A total of 28 such patients were thus enrolled in the study. Patients with Pemphigus and Bullous Pemphigoid disorders and age greater than 18 years were included in the study those who are unwilling to participate in the study were excluded. Permission for checking OP records and IP records of patients was obtained from the hospital authorities through proper channel. Direct interview with the patient using valid questionnaires.

\section{Study instruments}

- Standard data collection form

- "Morisky Green Levine Medication Adherence Scale questionnaire"

- “Dermatology Life Quality Index” (DLQI) Permission for using DLQI was obtained by proper channel.

- Modified Kuppuswamy’s socio-economic scale 


\section{Study Procedure}

All patients who satisfied the inclusion criteria were selected for the study. Detailed information regarding the study was described to the participants and obtained the informed consent. Information regarding age, gender, comorbidities and drugs used were obtained by referring their case sheet. Medication adherence was calculated by using "Morisky Green Levine medication adherence scale". Quality of life was assessed by DLQI. The initial Quality of Life DLQI 1 and at 3 months of follow up (DLQI 2) was assessed and the difference between the two was also analyzed in terms of medication adherence.

\section{Ethical consideration}

Institutional Human Ethics Committee of the tertiary care center provided the ethical clearence. Ethics Committee Approval number: HEC No.11/24/2019/ MCT. All data were kept confidential to be used for the purpose of this study only.

\section{Statistical Analysis}

SPSS version 21 used for the analysis of data. Continuous variables were expressed as mean $\pm S D$. Unpaired $t$-test was employed to compare differences between the continuous variables. Correlation was used for finding relationship between quantitative variables.

\section{RESULTS}

The observations of the sociodemographic characters, clinical aspects and medication adherence in terms of the 28 patients with pemphigus or bullous pemphigoid participated in the study is given below.

\section{Socio-demographic characters}

The age of the patients varied from 24 to 82 , female to male ratio was $1: 1$, majority of the patients lived in the urban areas and majority were married. (Table 2) Most of the patients belonged to either the lower middle-income group or the upper lower income group (Figure 1).

\section{Clinical aspects}

There were more patients with bullous pemphigoid (60.7\%) than pemphigus group of disorders (Figure 2) and $60.7 \%$ patients received DCP therapy as compared to only $35.7 \%$ on corticosteroid therapy with supportive measures. (Figure 3).

Table 2: Distribution of sample according to Socio-demographic characters.

\begin{tabular}{ccccc} 
Age & Gender & Residential area & Marital status & Comorbidities \\
& & & & \\
\hline Mean:52.61 & Male $: 50 \%$ & Rural: $35.7 \%$ & Married $: 85.7 \%$ & HTN $: 32.1 \%$ \\
Minimum:24 & Female:50\% & Urban:64.3\% & Unmarried:7.1\% & DM :39.3\% \\
Maximum:82 & & Widowed: $: 1.1 \%$ & DLP : $14.3 \%$ & Drug Allergy :0.0\% \\
& & & & Others :28.6\% \\
& & & & \\
\hline
\end{tabular}

DM: Diabetes mellitus, HTN: hypertension, DLP: dyslipidaemia

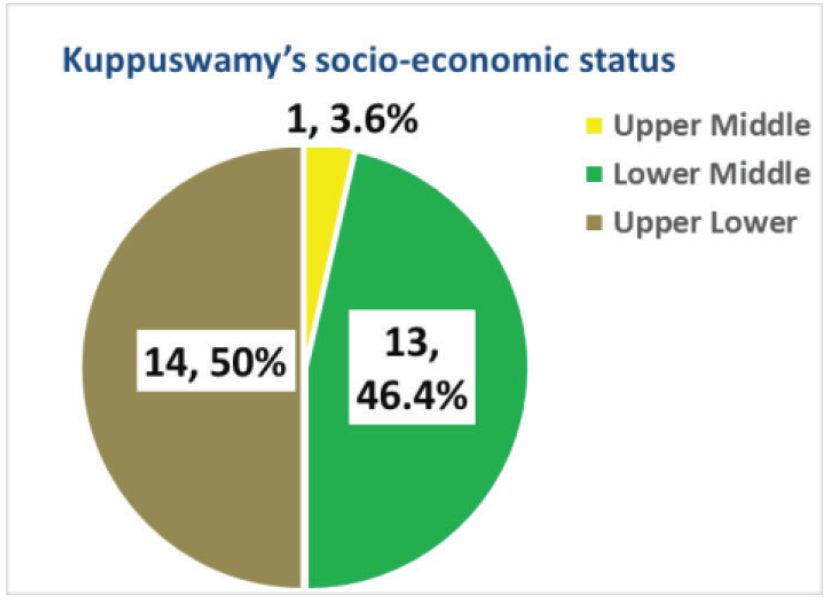

Figure 1: Distribution of Sample According to Socioeconomic Status.

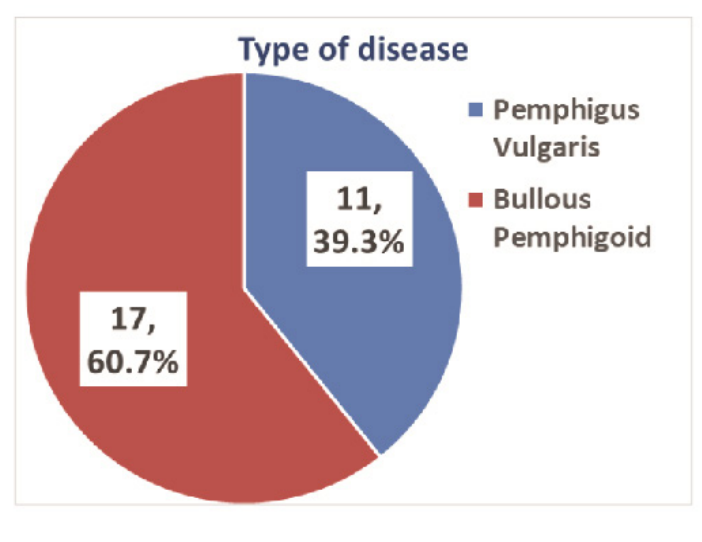

Figure 2: Distribution of Sample According to type of Disease. 


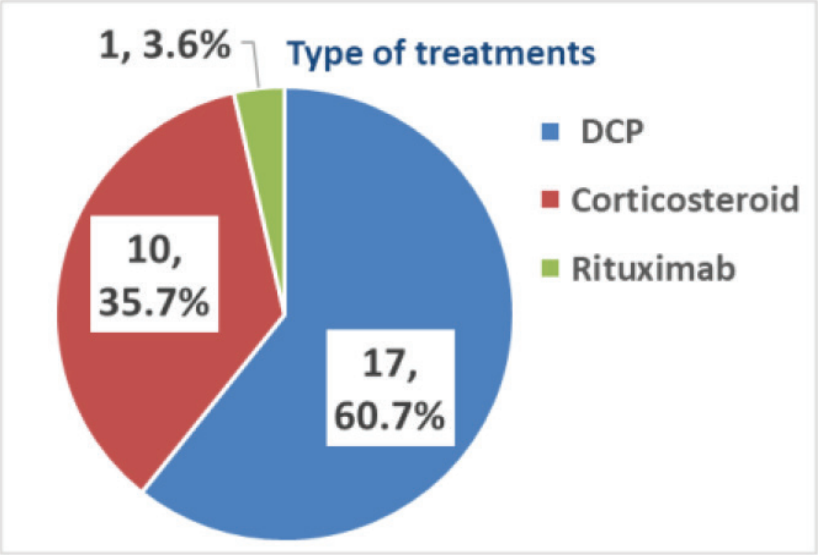

Figure 3: Distribution of Sample According to Type of Treatment.

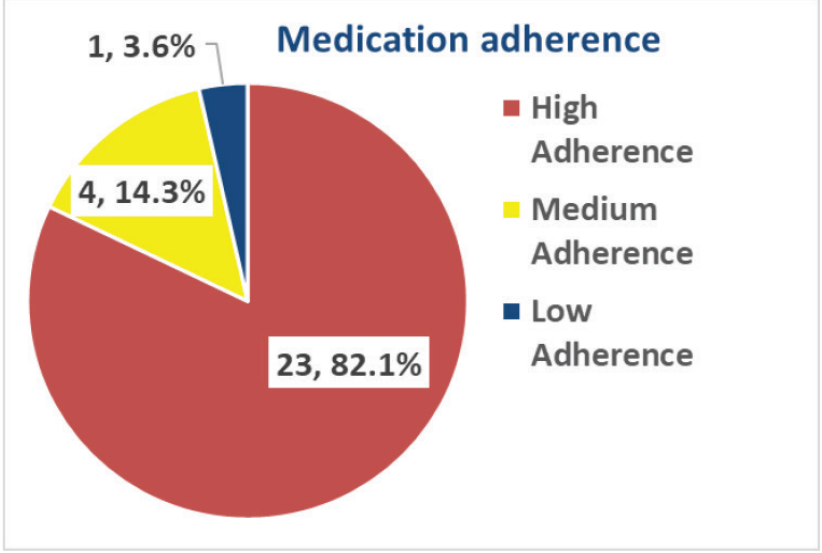

Figure 4: Distribution of Sample According to Medication Adherence.

\section{Medication adherence among patients}

In this study, levels of medication adherence calculated as per the "Morisky Green Levine medication adherence scale" is shown in (Figure 4).

Most patients at no time forgot to take their medicine nor were they even occasionally careless about taking their medicines. They did not at all stop the medications they were on, even when they felt better.

Majority of patients (82.1\%) showed High Adherence to therapy. $14.3 \%$ of patients showed Moderate adherence and only $3.6 \%$ of patients found to be Low medication adherence group.

\section{Quality of life}

The initial QoL of patients, DLQI 1, had a mean score of 14.11 \pm 4.508 . DLQI 2, which is the QoL of the same patients after 3 months (follow -up) had a mean score of $4.86 \pm 4.897$. (Table 3 ).

\section{Table 3: DLQI 1 and DLQI 2.}

\begin{tabular}{cccc}
\hline DLQI & Mean & N & SD \\
\hline DLQI 1 & 14.11 & 28 & 4.50 \\
DLQI 2 & 4.86 & 28 & 4.89 \\
\hline
\end{tabular}

N: Number, DLOI 1: Initial quality of life, SD: standard deviation, DLOI 2: Quality of life after 3 months

Table 4: Comparison between DLQI 1 and DLQI 2.

\begin{tabular}{|c|c|c|c|c|c|c|c|}
\hline \multicolumn{5}{|c|}{ Paired Differences } & \multirow{3}{*}{\multicolumn{2}{|c|}{$T \quad$ df }} & \multirow{3}{*}{$\begin{array}{c}\text { Significance } \\
\text { (2-tailed) }\end{array}$} \\
\hline \multirow[t]{2}{*}{ Mean } & \multirow[t]{2}{*}{ SD } & \multirow[t]{2}{*}{ SEM } & \multicolumn{2}{|c|}{$\begin{array}{l}95 \% \text { Confidence } \\
\text { Interval of the } \\
\text { Difference }\end{array}$} & & & \\
\hline & & & Lower & Upper & & & \\
\hline 9.25 & 5.67 & 1.07 & 7.05 & 11.45 & 8.62 & 27 & $<0.001$ \\
\hline
\end{tabular}

QoL: Quality of life, SEM: Standard error mean, N: Number, SD: standard deviation

\section{Table 5: Medication Adherence v/s DLQI Difference.}

\begin{tabular}{ccccc}
$\begin{array}{c}\text { Medication } \\
\text { adherence }\end{array}$ & $\mathbf{N}$ & $\begin{array}{c}\text { Mean } \\
\text { QoL }\end{array}$ & SD & SEM \\
High Adherence & 23 & 11.04 & 4.405 & 0.91 \\
Medium Adherence & 4 & 1.25 & 2.98 & 1.49 \\
Low Adherence & 1 & 0.00 & 0.00 & 0.00 \\
\hline
\end{tabular}

$D f$ : degrees of freedom, $T$ : $t$ value

In this study, there is a statistically significant difference between DLQI 1 and DLQI 2 of patients with a $p$ value $<0.001$. (Table 4).

\section{Medication Adherence v/s quality of life Difference}

From the study, Medication Adherence showed a statistically significant relationship with QoL. This result indicated that when Medication Adherence improved, the QoL also increased. There was a proportional increase in the quality of life. The difference in quality of life of high adherence group and low adherence group was found to be statistically significant $(p=0.023)$. Similarly, while comparing the Difference in QoL of high adherence group with medium adherence group, it also showed a statistically significant difference with a p value of $<0.001$. (Table 5,6 )

\section{DISCUSSION}

From our results, most of patients (82.1\%) had a High Adherence to the medication given for treatment of their skin disorder, pemphigus or bullous pemphigoid. About $14.3 \%$ of patients had Moderate adherence to medication and only $3.6 \%$ of patients comes under Low medication adherence group. The initial quality of life of these patients at the beginning of the study, with a mean score 
Table 6: Comparison of difference in quality of life.

\begin{tabular}{cccccccc}
\hline Comparing the quality of life & T & df & $\begin{array}{c}\text { Significance } \\
\text { (2-tailed) }\end{array}$ & $\begin{array}{c}\text { Mean } \\
\text { Difference }\end{array}$ & $\begin{array}{c}\text { Std. Error } \\
\text { Difference }\end{array}$ & $\begin{array}{c}\text { 95\% Confidence } \\
\text { Interval of the } \\
\text { Difference } \\
\text { Lower }\end{array}$ \\
$\begin{array}{c}\text { Upper } \\
\text { high adherence and low adherence }\end{array}$ & 2.45 & 22 & 0.023 & 11.04 & 4.50 & 1.71 & 20.37 \\
high adherence and medium adherence & 4.24 & 25 & $<0.001$ & 9.79 & 2.30 & 5.04 & 14.54 \\
\hline
\end{tabular}

$D f:$ degrees of freedom, $T: t$ value

of $14.11 \pm 4.508$ and the second follow-up assessment after 3 months, with a mean score of $4.86 \pm 4.897$, showed a statistically significant difference with $p$ value of $<0.001$. Details of any reports in the literature of similar studies which assessed Medication adherence in these bullous disorders using the MGLS or its relation to quality of the patient's life was not available, to make a comparison. However, a retrospective study of 245 cases of Pemphigus vulgaris over 10 years done by assessing adherence to medication as the ability of the patient (evaluated as a percentage) to attend clinic appointments as scheduled, and take medication as prescribed by the clinician at each visit showed a mean overall adherence of $55.8 \pm 17.21 \% .{ }^{13}$ The study concluded that the low adherence directly influenced the development of complications related to the disorder or treatment.

Another retrospective study of 45 patients aged 41-60 years, with autoimmune vesiculobullous disorders (AIVBD) over 5 years (2012-2017) was done by telephonic interview using a self-made questionnaire. ${ }^{14}$ Patients who had stopped medication for at least 1 week or more continuously during their treatment period were considered as nonadherent. Females outnumbered males (28:17). The commonest disorder was pemphigus vulgaris (57.78\%) followed by bullous pemphigoid (31.1\%) and epidermolysis bullosa aquisita (2 cases) and one chronic bullous disease of childhood. From the 26 patients with Pemphigus vulgaris, $69.23 \%$ showed adherence to medications and while both the cases of pemphigus foliaceous were non adherent. Among 14 cases of bullous pemphigoid, 78.57\% were adherent and 3 non-adherent. Among the total AIVBD patients, 64.4\% showed adherence to treatment and $35.5 \%$ were nonadherent but the grading into high, medium or low adherence was not done in that study. In this study, adherence to medication showed a statistically significant relationship with difference in QoL. This indicates that medication adherence is directly proportional to QoL of patients. A statistically significant difference was observed between the difference in QoL of High adherence group and Low Adherence group $(p=0.023)$ as well as that of the
High adherence group and Medium Adherence groups $(p<0.001)$. Medication adherence is very important for management of all disorders, especially chronic disorders. Poor adherence to the medication can cause serious health issues. ${ }^{15}$ Health care providers such as doctors, pharmacists and nurses have an important to improve medication adherence of patients.

Barriers against good medication adherence could be based on multiple factors relating to the patient, care provider or the health system. Factors like financial issues, comorbidities, false beliefs and superstitions etc are reasons for lack of adherence. To medications. Patients with chronic conditions and low literacy which causes difficulty in reading and understanding instructions may have lesser adherence to medication. ${ }^{16,17}$

Identifying the specific barriers for each patient and implementing appropriate methods to overcome them will be essential to enhance the medication adherence. Since pharmacists have direct access to patients in handing out medication, they are in an advantageous position to identify poor adherence and also the factors causing non-adherence and can help remove these barriers. Hence, pharmacists have a significant role to help patients maintain adherence to medication.

Patients should be educated about the necessity of adherence and may be helped by methods to help with adherence using medication aids like pillboxes and dosing calendars and avoiding running out of medications by refill reminders by the pharmacist.

\section{CONCLUSION}

The majority $(82.14 \%)$ of patients with autoimmune bullous disorders Pemphigus and Bullous Pemphigoid in this study, showed a high adherence to treatment having never stopped medications even when they felt better. Only as low as 3.6\% showed low medication adherence. There was a statistically significant difference in the QoL at the start of treatment and follow -up stage. 
The QoL at start and after 3 months (follow -up) was $14.11 \pm 4.508$ and $4.86 \pm 4.897$, respectively.

Medication Adherence showed a statistically significant relationship with quality-of-life difference. This indicates Medication adherence is directly proportional to QoL of patients. There was also a statistically significant difference between the difference in quality of life of High Adherence group and Low Adherence group as well as that of the High adherence group with Medium Adherence group. Literature search has not brought out similar studies in these bullous disorders.

Since adherence to treatment plays a vital role in management of diseases especially chronic and lifethreatening disorders like pemphigus and bullous pemphigoid, and because of its impact on the healthcare system, patients should be educated on the importance of medication adherence. Ways and means to help with medication adherence should be identified for the individual patient. It is very important to realise that the pharmacist has a key role to play in this regard.

\section{LIMITATIONS}

The information was collected from a single centre and could be conducted for a short duration only due to COVID-19 restrictions for patients.

\section{ACKNOWLEDGEMENTS}

We are extremely grateful to Dr. Sankar Sethuraman, Department of Community Medicine, and to all doctors, nurses and other staff members of Department of Dermatology and Venereology.

\section{CONFLICT OF INTEREST}

The authors declare that they have no conflicts of interest.

\section{ABBREVIATIONS}

MGLS: Morisky Green Levine Medication Adherence Scale; QoL: Quality of Life; DLQI: Dermatology Life Quality Index; DCP: Dexamethasone cyclophosphamide pulse.

\section{SUMMARY}

Pemphigus and Bullous Pemphigoid influence QoL of patients. Management of these chronic condition require different modes of long duration treatments. Therefore, medication adherence has a very important and essential role in treating these disorders. In this study Majority of patients have high medication adherence, there for the quality of life such patients also found to be good.

\section{REFERENCES}

1. Dobbels F, Van Damme-Lombaert R, Vanhaecke J, De Geest S. Growing pains: non-adherence with the immunosuppressive regimen in adolescent transplant recipients. Pediatr Transplant. 2005;9(3):381-90. doi: 10.1111/j.13993046.2005.00356.x, PMID 15910397.

2. Spiro H. Compliance, adherence, and hope. J Clin Gastroenterol. 2001;32(1):5. doi: 10.1097/00004836-200101000-00003, PMID 11154171.

3. DiMatteo MR. Adherence to treatment. In: Feldman MD, Christensen JF, editors, Editors Behavioral medicine in primary care. A practical guide. Stamford, CT: Appleton \& Lange; 1997. p. 136-40.

4. Roter DL, Hall JA, Merisca R, Nordstrom B, Cretin D, Svarstad B. Effectiveness of interventions to improve patient compliance: a meta-analysis. Med Care. 1998;36(8):1138-61. doi: 10.1097/00005650-199808000-00004, PMID 9708588

5. Segeral O, Madec Y, Ban B, Ouk V, Hak CR, Le Tiec C, Nerrienet E, Goujard C, Taburet AM, Delfraissy JF, Fontanet A. Simplified assessment of antiretroviral adherence and prediction of virological efficacy in HIV-infected patients in Cambodia. AIDS Res Treat. 2010;2010:142076. doi: 10.1155/2010/142076.

6. Beyhaghi H, Reeve BB, Rodgers JE, Stearns SC. Psychometric properties of the four-Item Morisky Green Levine medication adherence scale among atherosclerosis risk in communities (ARIC) study participants. Value Health. 2016;19(8):996-1001. doi: 10.1016/j.jval.2016.07.001, PMID 27987650.

7. Morisky DE, Green LW, Levine DM. Concurrent and predictive validity of a selfreported measure of medication adherence. Med Care. 1986;24(1):67-74. doi: 10.1097/00005650-198601000-00007, PMID 3945130.

8. Wang Y, Lee J, Toh MP, Tang WE, Ko Y. Validity and reliability of a selfreported measure of medication adherence in patients with type 2 diabetes mellitus in Singapore. Diabet Med. 2012;29(9):e338-44. doi: 10.1111/j.14645491.2012.03733.x, PMID 22672497.

9. Koschack J, Marx G, Schnakenberg J, Kochen MM, Himmel W. Comparison of two self-rating instruments for medication adherence assessment in hypertension revealed insufficient psychometric properties. J Clin Epidemiol. 2010;63(3):299-306. doi: 10.1016/j.jclinepi.2009.06.011, PMID 19762213.

10. Finlay AY, Khan GK. Dermatology Life Quality Index (DLQI)—a simple practical measure for routine clinical use. Clin Exp Dermatol. 1994;19(3):210-6. doi: 10.1111/j.1365-2230.1994.tb01167.x, PMID 8033378.

11. Pouran L, Yalda N, Iman M, Mohammad Taghi S. Quality of life evaluation in patients with pemphigus vulgaris. Iran J Dermatol. 2013;16(3):100-4.

12. Wani RT. Socioeconomic status scales-modified Kuppuswamy and Udai Pareekh's scale updated for 2019. J Family Med Prim Care. 2019;8(6):1846-9. doi: 10.4103/jfmpc.jfmpc_288_19, PMID 31334143.

13. Tirado-Sánchez A, Bonifaz A, Ponce-Olivera RM, Espinosa-Díaz S, VazquezGonzález D. Prevalence of adherence to dermatological treatments among patients with pemphigus vulgaris and its relationship with complications and death: a single-center, cross-sectional study. Research. 2014;1:723. doi: 10.13070/rs.en.1.723.

14. Ajmal KV, Priya P, Asokan N. Drug adherence in patients with autoimmune vesiculobullous disorders-a cross sectional study. Int $\mathrm{J}$ Dermatol. 2020;59(2):41-3.

15. Anon. Poor medication adherence increases healthcare costs. Pharmacoecon Outcomes News. 2005;480:5.

16. Osterberg L, Blaschke T. Adherence to medication. N Engl J Med. 2005;353(5):487-97. doi: 10.1056/NEJMra050100, PMID 16079372.

17. Praska JL, Kripalani S, Seright AL, Jacobson TA. Identifying and assisting lowliteracy patients with medication use: a survey of community pharmacies. Ann Pharmacother. 2005;39(9):1441-5. doi: 10.1345/aph.1G094, PMID 16046489. 